\title{
Dirac Equation in Cosmological Inertial Frame
}

\author{
Sangwha-Yi*
}

Department of Math, Taejon University 300-716, South Korea

*Corresponding Author: Sangwha-Yi, Department of Math, Taejon University 300-716, South Korea

Abstract: Dirac equation is a one order-wave equation. Wave function uses as a probability amplitude in quantum mechanics. We make Dirac Equation from wave function, Type A in cosmological inertial frame. The Dirac equation satisfy Klein-Gordon equation in cosmological inertial frame.

Keywords: Gordon Equation; Cosmological Inertial Frame;Dirac equation;

PACS Number: 03.30. $+p, 03.65$

\section{INTRODUCTION}

Dirac equation is in special relativity theory,[8]

$\left(i \hbar \gamma^{\mu} \partial_{\mu}-m c I\right) \psi=0$

$I$ is $4 \times 4$ unit matrix,

$\gamma^{0}=\left(\begin{array}{cc}I^{\prime} & 0 \\ 0 & -I^{\prime}\end{array}\right), \quad \gamma^{i}=\left(\begin{array}{cc}0 & \sigma^{i} \\ -\sigma^{i} & 0\end{array}\right), \quad \sigma^{1}=\left(\begin{array}{cc}0 & 1 \\ 1 & 0\end{array}\right), \sigma^{2}=\left(\begin{array}{cc}0 & -i \\ i & 0\end{array}\right), \sigma^{3}=\left(\begin{array}{cc}1 & 0 \\ 0 & -1\end{array}\right)$

$I^{\prime}$ is $2 \times 2$ unit matrix, $\sigma^{i}$ is Pauli's matrix.

\section{Dirac Equation from WaVe FunCtion-TyPe A in CoSmological Inertial Frame}

Dirac equation is the wave equation. Therefore, Dirac equation is in cosmological inertial frame,[2]

Wave function Type A:

$r \rightarrow r \sqrt{\Omega\left(t_{0}\right)} \quad, \quad t \rightarrow \frac{t}{\sqrt{\Omega\left(t_{0}\right)}}$,

$t_{0}$ is the cosmological time. $\Omega\left(t_{0}\right)$ is the expanding ratio of universe in the cosmological time $t_{0}$.

$\left(i \hbar \sqrt{\Omega\left(t_{0}\right)} \gamma^{0} \partial_{0}+i \hbar \frac{1}{\sqrt{\Omega\left(t_{0}\right)}} \gamma^{i} \partial_{i}-m c I\right) \phi=0$

If $\bar{\partial}_{\mu}$ is

$\bar{\partial}_{\mu}=\left(\sqrt{\Omega\left(t_{0}\right)} \partial_{0}, \frac{1}{\sqrt{\Omega\left(t_{0}\right)}} \partial_{i}\right)$

Dirac equation is in cosmological inertial frame,

$\left(i \hbar \gamma^{\mu} \bar{\partial}_{\mu}-m c I\right) \phi=0$

Eq(4) multiply $i \hbar \gamma^{v} \bar{\partial}_{v}$, hence 
$\left(-\hbar^{2}\left(\gamma^{\mu} \bar{\partial}_{\mu}\right)\left(\gamma^{v} \bar{\partial}_{v}\right)-i \hbar\left(\gamma^{v} \bar{\partial}_{v}\right) m c I\right) \phi=0$

In this time,

$$
i \hbar \gamma^{v} \bar{\partial}_{v} \phi=m c I \phi
$$

Hence, $\mathrm{Eq}(5)$ is

$$
\left(-\hbar^{2} \gamma^{\mu} \gamma^{v} \bar{\partial}_{\mu} \bar{\partial}_{\nu}-m^{2} c^{2} I\right) \phi=0
$$

In this time, matrix $\gamma^{\mu}$ is

$$
\frac{1}{2}\left(\gamma^{\mu} \gamma^{v}+\gamma^{v} \gamma^{\mu}\right)=\frac{1}{2}\left\{\gamma^{\mu}, \gamma^{\nu}\right\}=\eta^{\mu \nu} I
$$

Therefore,[1],[3]

$$
\begin{aligned}
& \frac{1}{2}\left(\gamma^{\mu} \gamma^{\nu}+\gamma^{v} \gamma^{\mu}\right) \bar{\partial}_{\mu} \bar{\partial}_{\nu} \phi+\frac{m^{2} c^{2}}{\hbar^{2}} I \phi \\
& =\left(\eta^{\mu \nu} \bar{\partial}_{\mu} \bar{\partial}_{\nu}+\frac{m^{2} c^{2}}{\hbar^{2}}\right) I \phi=0
\end{aligned}
$$

$\mathrm{Eq}(9)$ is the matrix equation of Klein-Gordon.

Dirac spinor $\phi$ is $\phi=\left(\phi_{1}, \phi_{2}, \phi_{3}, \phi_{4}\right)$. $\phi$ 's hermitian conjugate $\phi^{+}=\left(\phi_{1}^{*}, \phi_{2}^{*}, \phi_{3}^{*}, \phi_{4}^{*}\right)$.

Hence, $\phi$ 's adjoint spinor $\bar{\phi}$ is

$$
\bar{\phi}=\phi^{+} \gamma^{0}, \bar{\phi}\left(i \gamma^{\mu} \bar{\partial}_{\mu}+m c I\right)=0
$$

Hence, positive probability density $j^{0}$ is

$$
j^{0}=\bar{\phi} \gamma^{0} \phi=\phi^{+} \phi=\left|\phi_{1}\right|^{2}+\left|\phi_{2}\right|^{2}+\left|\phi_{3}\right|^{2}+\left|\phi_{4}\right|^{2}
$$

\section{CONCLuSion}

We found Dirac equation from Wave Function-Type A in cosmological special theory of relativity. The wave function uses as a probability amplitude.

\section{REFERENCES}

[1]S.Yi, "Klein-Gordon Equation and Wave Function in Cosmological Special Theory of Relativity", International Journal of Advanced Research in Physical Science,7,12(2020),pp4-6

[2]S.Yi, "Yukawa Potential in Klein-Gordon Equation in Cosmological Inertial Frame", International Journal of Advanced Research in Physical Science,8,3(2021),pp16-18

[3]S.Yi, "Cosmological Special Theory of Relativity" International Journal of Advanced Research in Physical Science, 7,11(2020),pp4-9 
[4]A.Beiser,"Concepts of Modern Physics"4th Edition,( Mcgraw-Hill,1994)

[5]J.D. Bjorken \& S. D. Drell, Relativistic Quantum Field(McGraw- Hill Co., 1965)

[6]P.Bergman,Introduction to the Theory of Relativity(Dover Pub. Co.,Inc., New York,1976), Chapter V

[7]R.L.Liboff, Quantum Mechanics(Addison-Wesley Publishing Co., Inc.,1990)

[8]A.Beiser, Concept of Modern Physics(McGraw-Hill,Inc.,1991)

Citation: Sangwha-Yi (2021) Dirac Equation in Cosmological Inertial Frame. International Journal of Advanced Research in Physical Science (IJARPS) 8(7), pp.4-6, 2021.

Copyright: () 2021 Authors, This is an open-access article distributed under the terms of the Creative Commons Attribution License, which permits unrestricted use, distribution, and reproduction in any medium, provided the original author and source are credited. 